\title{
I know nothing
}

\author{
Derek Richards \\ Editor, Evidence-Based Dentistry
}

Evidence-Based Dentistry (2003) 4, 47. doi:10.1038/sj.ebd.6400215

In this issue of Evidence-Based Dentistry we look at a number of systematic reviews published as the result of an initiative by the European Federation of Periodontology. These represent a commitment by the Federation and its members to clarify the evidence-base of periodontology. The production of these reviews - as with any systematic review - represents a great deal of work. This is not just in terms of the review itself but also the training necessary to bring all the participants up to speed.

The results summarised in the journal have also been produced over a relatively short timescale for systematic reviews (less than a year). This owes much to the enthusiastic core group of authors and the support of Ian Needleman and David Moles, who are the Director and Deputy Director respectively of the International Centre for Evidence-based Periodontology, based at the Eastman Dental Institute in London, UK.

The reviews provide answers to some questions that periodontologists have been debating for some time. Nevertheless, as with most reviews that have been completed in dentistry to date, they leave us with further questions remaining to be resolved. Even in the case of periodontology, which has been leading dentistry in the number of randomised controlled trials it produces, the review teams found few trials of good quality and comparable outcomes, limiting their ability to combine studies. There are a variety of reasons for this, and there is a need for the dental research community to address these issues, and not just in periodontal studies.

Many people question the value of systematic reviews because they often seem to be inconclusive. This is a negative interpretation of the valuable role they perform. Systematic reviews locate, appraise and synthesise evidence and thus provide a valuable benchmark of the current state of the dental evidence. They are a retrospective exercise of necessity, and they can only summarise the evidence that is available. If researchers do not like the message, "don't shoot the messenger". Clarifying dental evidence is a primary role of the systematic review: in doing this such reviews inform us of the strength of evidence on which our current practice is based (good or bad), and any potential negative effect of treatment. They also identify obvious knowledge gaps requiring future research. All of these features are important to practitioners and to those who provide services, researchers and funding, as well as to patients.

In his text book Evidence-based Health Care, ${ }^{1}$ Muir Gray categorised three types of treatment and their impact on patients: those that do more good than harm; those that do more harm than good; and those of unknown effect. The number of treatments that have good evidence that they do more good than harm in dentistry is limited and I would suggest that the majority currently fall into the category of unknown effect. I imagine that many members of the dental profession today believe that most of what they do is of benefit to their patients either in the short or long term. Perhaps the wisest amongst us should realise that, as Socrates suggested, we must first accept that we know very little about the effectiveness of our treatments.

This issue of $E B D$ also outlines the career of Helen Worthington, who has been made Professor of Evidence-based Care at Manchester University. This honour is richly deserved because Helen is one of the unsung driving forces behind the Cochrane Oral Health Group. I would like to add my personal congratulations on her appointment. Helen is one of those rare resources within dentistry in the UK: a dental statistician. Perhaps her appointment will lead to more statistical input in dental research within other dental research groups in the UK - preferably at the study design stage rather than just after the data collection has been completed. Perhaps then we can begin to address some of the quality issues within study design in dentistry.

1. Muir Gray JA. Evidence-based Health Care. London: Churchill Livingstone; 1997. 